Tohoku J. exp. Med., 1964, 84, 259-273

\title{
On Mechanism of Coagulation and Solidification of Gallstone Ingredients in Bile
}

By

\author{
Tetsuo Maki and Noriyoshi Suzuki \\ From the Department of Surgery, Tohoku University School of \\ Medicine, Sendai; Director: Prof. T. Maki \\ (Received for publication, July 17, 1964)
}

\begin{abstract}
Although a number of theories are available on the mechanism of separation of gallstone ingredients from bile, nothing is practically known as to how the separated ingredients coagulate and solidify in bile to form gallstones. This paper presents a theory on coagulation in bile of gallstones on the basis of a series of experimental facts. An outline of the theory is as follows: The gallstone ingredients separated from bile first form stable lyophobic colloids, but the stability of the colloids is soon decreased by effects of inorganio electrolytes (electrostatic effect) and high-molecular-weight organic substances (electrostatic effect and bridging action) of bile and the colloids are deposited as flocks. The flocks then gradually solidify under the influence of various types of kinetic energies that are exerted by the organism and are ultimately transformed into solid gallstones. In support of this theory, gallstone-like concrements were actually produced in vitro using a suspension of human bile sediment consisting mainly of calcium bilirubinate. This theory may also be applicable, to some extent, to the coagulation mechanism of other substances separated from a colloidal solution in the living organism.
\end{abstract}

In proportion to the recent progress of assay methods in the field of biological chemistry, many research works have dealt with chemical constitution of bile and gallstones, and the mechanism of separation of gallstone constituents from bile has become more and more precisely clarified. However, the mechanism how the gallstone constituents coagulate and solidify in bile, that is a colloidal solution, to form a solid concrement still remains completely obscure.

As regards calcium bilirubinate stones or so-called pigmented calcium stones, a series of experimental studies at the authors' department have elucidated that calcium bilirubinate, the main constituent of this type of gallstone, is formed in infected bile by the enzymic activity of bacterial $\beta$-glucuronidase ${ }^{1)}$ and that the sediment appearing in infected bile is almost identical in chemical composition. with a calcium bilirubinate stone $\mathrm{e}^{2)}$.

In this connection, the authors have investigated the theoretical basis for

䫐 哲夫，鉿木範美 
coagulation and solidification of such a bile sediment and have succeeded in experimentally producing a gallstone-like concrement, in vitro, from a suspension of human bile sediment consisting chiefly of calcium bilirubinate. Under the belief that this fact would explain, to a considerable extent, the mechanism of gallstone formation in vivo, the experimental results are reported below.

\section{MATERIAL AND METHODS}

\section{Material}

Bile specimens were obtained, through a $\mathrm{T}$ tube inserted in the common bile duct, from patients with calcium bilirubinate stones in which bile was infected mostly with Escherichia coli. The specimens were pooled and incubated on water bath for 48 hours at $38^{\circ} \mathrm{C}$. The sediment that appeared after incubation was then isolated by centrifuging and washed 10 times with distilled water. The final sediment was suspended into distilled water so that each milliliter of the suspension contained $22 \mathrm{mg}$ solid substance and this suspension served as the stock sample for the subsequent experiments. The specific conductivity of the stock sample measured approximately $2.1 \times 10^{-5} Q^{-1} \cdot \mathrm{cm}^{-1}$ when diluted $1: 10$ with distilled water.

\section{Methods}

Estimation of $\zeta$-potential of the sample: For estimating $\zeta$-potential of the sample, a portion of the stock sample was diluted 1:10 with distilled water and the electrophoretic mobility of the particles of the suspension was measured with a microscopic electrophoresis apparatus ${ }^{3)}$. The $\zeta$-potential of colloid particles, in general, can be estimated from the electrophoretic mobility of the particles by means of Smoluchowski's equation:

$$
\zeta=\frac{4 \pi \eta}{\varepsilon} \cdot u
$$

where $\eta$ denotes the coefficient of viscosity of the solution, $\varepsilon$ the dielectric constant of the solution, $H$ the gradient of the applied potential and $u$ the velocity of the particles. Since $H$ is expressed with the electric current $i$, the specific conductivity of the solution $\lambda$ and the cross sectional area of the electrophoresis cell $S$, as

$$
H=\frac{i}{\lambda S}
$$

the above equation can be transformed into:

$$
\zeta=\frac{4 \pi \eta S}{\varepsilon} \cdot \frac{\lambda}{i} \cdot u
$$

Substituting the numerical values for the present experimental condition $\varepsilon=80$, 
$\eta=0.01 \mathrm{dyne} \cdot \mathrm{cm}^{-2} \cdot \mathrm{sec}$ and $S=0.10 \mathrm{~cm}^{2}$, and measuring $\lambda$ in $\Omega^{-1} \cdot \mathrm{cm}^{-1}, i$ in $\mu \mathrm{A}$ and $u$ in $\mu \cdot \sec ^{-1}$, we obtain

$$
\zeta(\mathrm{mV})=1.41 \times 10^{6} \cdot \frac{\lambda}{i} \cdot u .
$$

This formula was utilized in numerical calculation of $\zeta$ of the sample. The specific conductivity $\lambda$ of the suspension was measured with an impedance bridge Type BV-Z-13B, Yokokawa Electric Co.

Evaluation of coagulating effect of various reagents on the sample suspension: To find out the substances that induce coagulation of the particles of bile sediment, a number of inorganic and organic reagents were tested for the effect on the sample suspension. Inorganic substances studied were sodium chloride, calcium chloride, aluminium chloride, precipitated calcium carbonate, hydrochloric acid and sodium hydroxide (analytical grade reagents of commercial supply), and the organic substances were sodium oleate, dodecyl ammonium acetate, gelatin, Separan 2610, Superfloc 16 (American Cyanamide Co.), Konanfloc 200, 1250 and 2000 (Konan Chemical Co.), and ZH (Konan Chemical Co.). The method of study was as follows: A $1 \mathrm{ml}$. (or $2.5 \mathrm{ml}$.) aliquot of the stock sample was put in a graduated glass cylinder of $10 \mathrm{ml}$. (or $25 \mathrm{ml}$.) capacity, a determined amount of the reagent to be tested was added and distilled water was poured into a total amount of $10 \mathrm{ml}$. (or $25 \mathrm{ml}$.). The mixture was then stirred gently with a glass rod and allowed to stand at room temperature. After 1 hour and 24 hours, the volume of sedimented flocks, if any, was measured and the turbidity of the supernatant was recorded on a five point scale: $-,+, H, H$ and $H+$, to represent the coagulating effect of the reagent.

Application of physical movement to the sample: A suspension of the sample was prepared in a $50 \mathrm{ml}$. Ellenmeyer flask with $2 \mathrm{ml}$. of the stock sample and an arbitrary amount of distilled water, to which were added pre-determined quantities of certain inorganic and organic substances that were revealed, in the above experiment, to have considerable coagulating effects. The total amount was then adjusted with distilled water to $32 \mathrm{ml}$. and to the mixture was supplied a kinetic energy by rotating the flask at 6 r.p.m. with a synchronous motor.

\section{RESULTS}

\section{Physico-chemical properties of the sample}

The sample prepared from sediments of infected bile resembled a calcium bilirubinate stone in the infrared absorption spectrum (Fig. 1). Hence, the sample was presumed to consist mostly of calcium bilirubinate. The sample was easily suspended into distilled water, yielding a suspension which was homogeneous in appearance and was quite stable unless other substances were added. The $\zeta$ potential of the suspending particles measured approximately $-20 \mathrm{mV}$ in distilled 


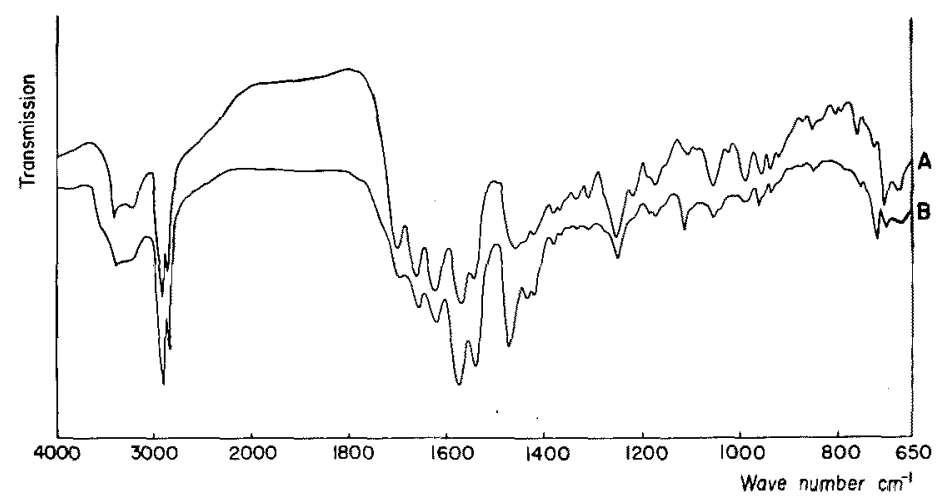

Fig. l. Infrared spectra of a calcium bilirubinate stone (A) and of the sediment obtained from bile that contained the stone (B). $\mathrm{KBr}$ disk method with a $\mathrm{NaCl}$ prism.

water. It was further confirmed that the particles of synthetic calcium bilirubinate which the authors had prepared ${ }^{4}$ also had negative charges when suspended into distilled water.

2. Coagulating effects of inorganic substances on the sample

Addition of inorganic electrolytes to the aqueous suspension of the sample induced a change in the $\zeta$-potential of the suspending particles and thus modified the stability of the suspension to various extent. Fig. 2 shows the sedimentation volume and the turbidity of the supernatant, after 1 hour, of the mixture containing $2.5 \mathrm{ml}$. of the stock sample and a various amount of an inorganic electrolyte in a total volume (with distilled water) of $25 \mathrm{ml}$. The change on this occasion in the $\zeta$-potential of the suspending particles is illustrated in Fig. 3, as a function of the concentration of the added electrolyte. Addition of sodium chloride resulted in a slow precipitation of the suspension; the sedimentation volume was small even after an hour and the supernatant remained quite turbid. When calcium chloride or aluminium chloride was added, on the other hand, precipitation progressed more rapidly, a larger amount of sediment was produced and the supernatant became relatively transparent.* The $\zeta$-potential of the particles became smaller on these occasions in the absolute value. However, when addition of aluminium chloride exceeded a certain level, a remarkable increase in the $\zeta$-potential to the positive side was observed and the particles were re-dispersed as evidenced by an increase in the turbidity of the supernatant. From these results, it may be concluded that inorganic cations, which are opposite in electric

* In a suspension or a lyophobic colloid solution, in general, there is a definite relation between the intensity of coagulation or the velocity of sedimentation and the sedimentation volume; the stronger the coagulation and the rapider the sedimentation, the larger is the sedimentation volume ${ }^{5}$. 


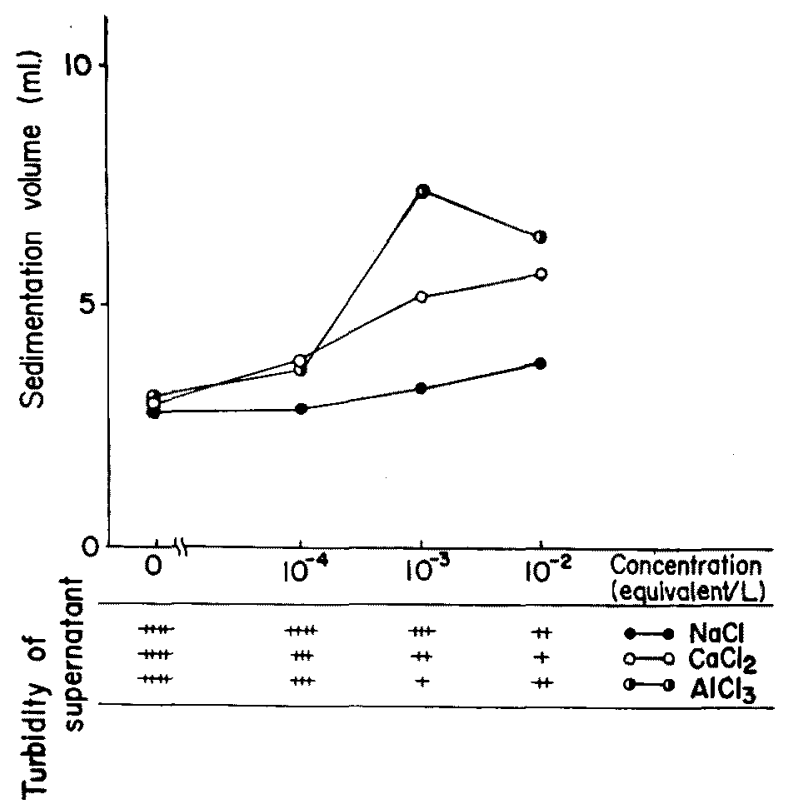

Fig. 2. Sedimentation volume and turbidity of the supernatant as functions of concentration of the inorganic electrolyte added to the suspension. One hour after addition of the electrolyte.

charge to the particles of bile sediment, induce coagulation of the latter particles and the larger the valence of the cation, the more remarkable the coagulating effect is. This agrees well with the fact known as the Schultz-Hardy's law.

The effect of $\mathrm{pH}$ on coagulation of the suspending particles of bile sediment was then studied by shifting $\mathrm{pH}$ of the suspension with hydrochloric acid or sodium hydroxide. In this case, sodium chloride was added to a concentration of 10 millimoles per liter of the suspension to serve as indifferent ions. The result is shown in Fig. 4. It may be seen that the absolute value of $\zeta$-potential of the particles decreased much and the particles coagulated remarkably when $\mathrm{pH}$ was shifted to the acidic side, while the $\zeta$-potential increased to the negative side and the suspension tended to disperse when $\mathrm{pH}$ was in an alkaline reaction. This fact implies that hydrochloric acid, though being a univalent electrolyte, has a considerable effect of lowering the $\zeta$-potential and promoting coagulation of the particles of bile sediment. It was also revealed in this series of experiment that the sample had buffering effects on hydrogen ions and hydroxide ions.

When coagulation occurred by addition of an inorganic electrolyte, the particles of bile sediment formed gel-like flocks which were quite hydrophilic and did not drain well, just like a deposit of iron hydroxide precipitated from an aqueous solution. Such a hydrophilic nature of the flocks is due to the presence 


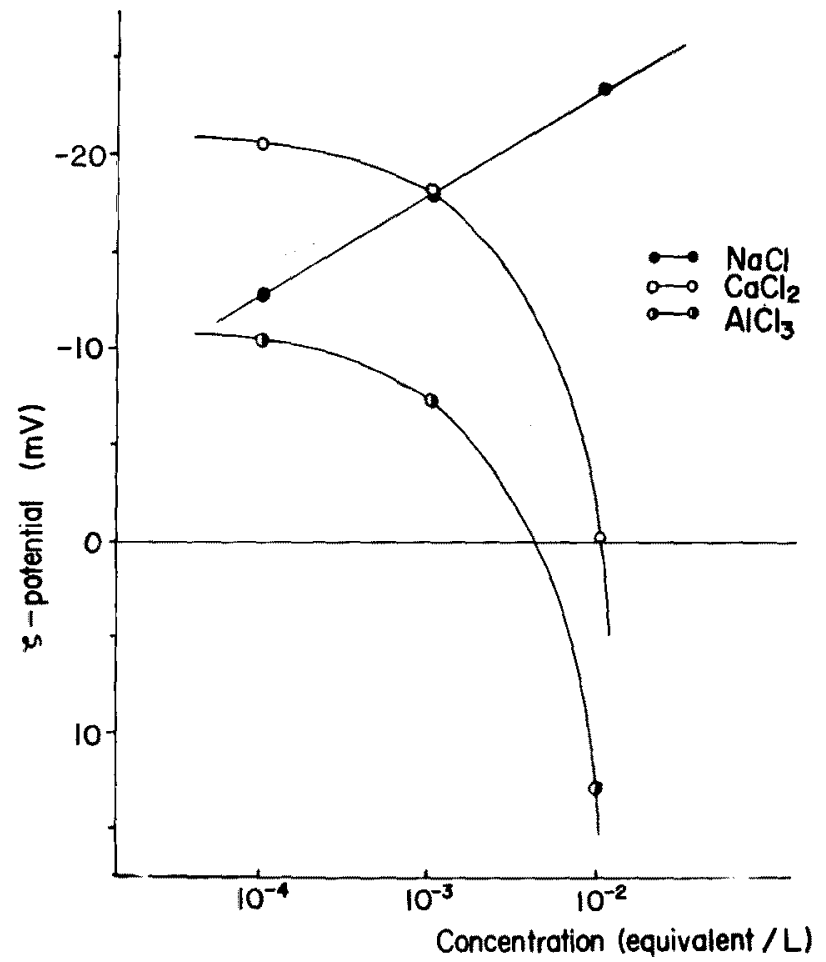

Fig. 3. 5-Potential of the particles as a function of concentration of the inorganic electrolyte added to the suspension.

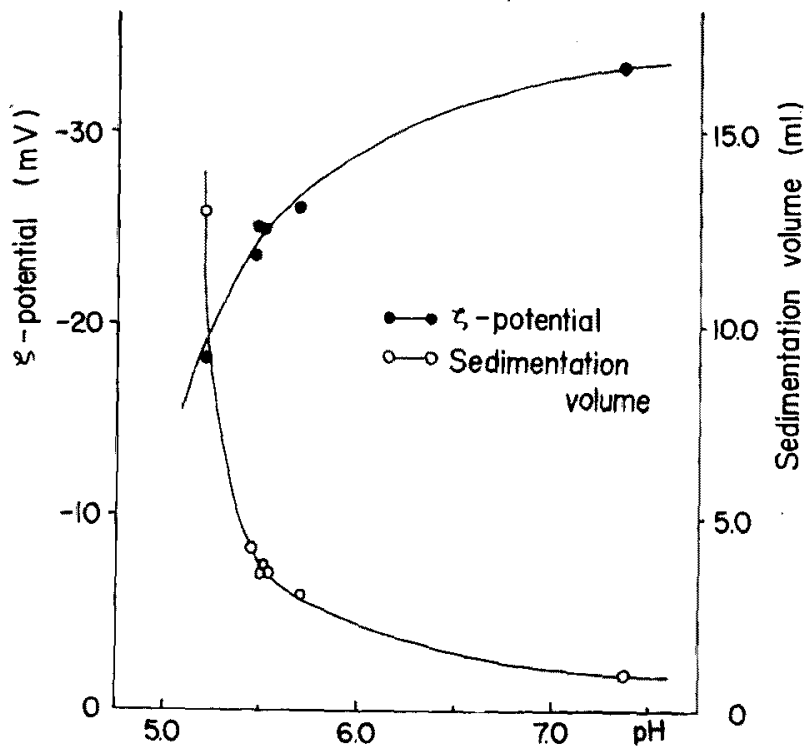

Fig. 4. Sedimentation volume and $\zeta$-potential of the particles as functions of $\mathrm{pH}$ of the suspension, in the presence of $10 \mathrm{mM} / \mathrm{L}$ of sodium chloride. 


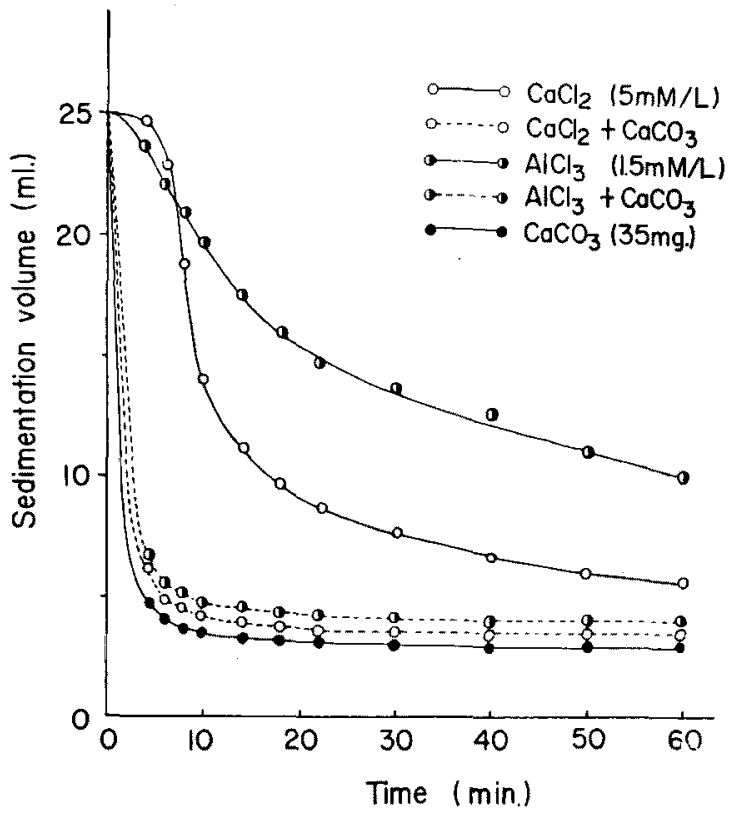

Fig. 5. Sedimentation rate of the suspension in the presence of calcium carbonate and inorganic electrolyte.

of a hydrated layer surrounding each of the particles, which must be removed to transform the flocks into well drained coagulates and further into gallstonelike solid concrements. Since calcium carbonate is known to improve considerably the precipitation characteristic of iron hydroxide ${ }^{6}$, this compound was then employed for studying the effect on the rate of sedimentation of the sample suspension. In Fig. 5 is shown the velocity of sedimentation of the particles of the sample by plotting the sedimentation volume (total amount of the suspension 25 $\mathrm{ml}$.) against time. Besides the curves for the suspensions containing calcium carbonate, the curves for those containing calcium chloride or aluminium chloride alone are also presented for comparison. The results show that by addition of calcium carbonate the rate of sedimentation is increased and the final volume of the sediment decreased, apparently indicating the effect of calcium carbonate to enhance sedimentation of the particles by an inorganic electrolyte and to dehydrate the sediment.*

3. Coagulating effects of organic substances on the sample

It has been known that a group of high-molecular-weight organic compounds

* On such occasion, the general rule between the intensity of coagulation and the sedimentation volume does not hold. 
are effective as coagulating agents for colloidal substances. In this connection, a number of such compounds were studied for the effects on the suspension of bile sediment, in the same manner as in the case for inorganic reagents. Of the high-molecular-weight organic compounds examined in this series, gelatin, Separan 2610, Superfloc 16, Konanfloc 200 and $\mathrm{ZH}$ revealed little or no effect to coagulate the sample suspension. On the other hand, Konanfloc 1250 and Konanfloc 2000, which are both linear polyelectrolytes of positive charge, exhibited marked coagulating effects. As an example, the effect of Konanfloc 2000 is shown in Fig. 6 which illustrates the sedimentation volume (total amount of the suspension $10 \mathrm{ml}$.) and the $\zeta$-potential of the suspending particles as functions of the concentration of this substance. The coagulation of the suspension as well as lowering of the $\zeta$-potential of the particles were remarkable even at a concentration as low as 0.5 per cent.

The above results indicate that a linear polyelectrolyte of positive charge has a particularly strong effect to coagulate the suspension of bile sediment. Subsequent experiments evidenced that the effect of such a polyelectrolyte was enhanced much by simultaneous addition of an inorganic electrolyte such as calcium chloride or aluminium chloride; on such occasions the coagulation of the particles was more remarkable and the supernatant became more transparent than in the suspension supplemented with a polyelectrolyte alone. Moreover, coagulation of the suspension was the most striking when a positive polyelectrolyte, an inorganic electrolyte and calcium carbonate were added altogether, the

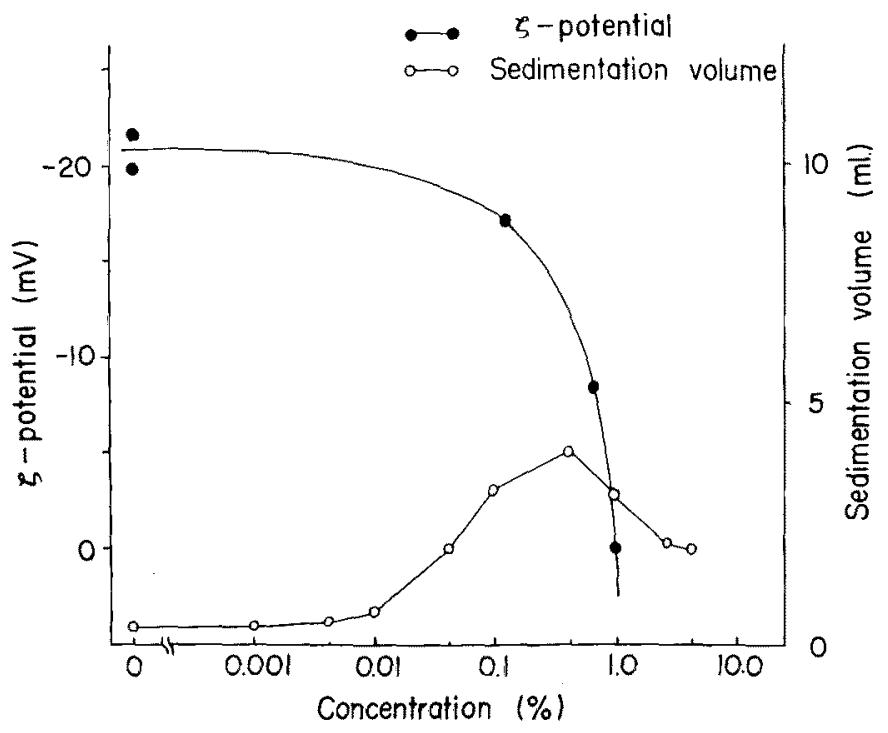

Fig. 6. Sedimentation volume and s-potential of the particles as functions of concentration of Konanfloc 2000 added to the suspension. 
rate of sedimentation being quite great and the sediment being relatively well dehydrated in this case.

Of comparatively low-molecular-weight organic compounds, sodium oleate and dodecyl ammonium acetate were also employed for studying the coagulating effect on the suspension of bile sediment. Sodium oleate did not coagulate but rather dispersed the suspension, although this compound exhibited an appreciable coagulating effect when an equivalent amount of hydrochloric acid was added, simultaneously. Dodecyl ammonium acetate coagulated the suspension but the effect was much less marked than those of the linear polyelectrolytes.

4. Results of application of physical movement to the sample suspension

In relation to the technique of preparing wet pellets of kaolinate, it has been recently pointed out by Yusa and Gaudin?) that a rotating movement of a suspension enhances coagulation of the suspending particles. On the basis of such a fact, the suspension of bile sediment was subjected to rotation with the inorganic and organic reagents that were effective for coagulation of the sample. In the course of rotation, visible coagulates were yielded in the suspension, and the coagulates grew up in size and finally formed gallstone-like solid concrements in about 30 minutes, the progress of the phenomenon depending upon the kind and amount of the reagents that were added to the system.

The coagulating agents used in this experiment were calcium carbonate, calcium chloride and Konanfloc 1250 or 2000 . Comparing the effect of Konanfloc 1250 with that of Konanfloc 2000 , it was found that the former compound produced solid coagulates of bile sediment at a concentration of 1 per cent or a little higher, while the latter produced larger coagulates more easily at a concentration around 0.15 per cent. Such a difference in the coagulating effect may probably be due to a difference in the grade of polymerization and in the characteristics of the functional groups of the molecule. It was also revealed that addition of Konanfloc 2000 and calcium chloride in this order yielded a more favorable result than in the case when they were added in the reversed order. Although the reason for this phenomenon is obscure, it is possible that an inorganic electrolyte not only decreases the $\zeta$-potential of the particles but also modifies favorably the fibrous structure of the linear polyelectrolyte that was adsorbed to the surface of the particles.

Fig. 7 shows the changes in $\zeta$-potential of the particles of the suspension at various combinations of calcium carbonate, Konanfloc 2000 and calcium chloride. When calcium carbonate only was added to the suspension, an appreciable coagulation occurred but the $\zeta$-potential remained almost unchanged. When the suspension was further supplemented with Konanfloc 2000, the absolute value of $\zeta$-potential decreased a little and coagulation became more remarkable. However, the decrease in the $\zeta$-potential as well as the intensity of coagulation 


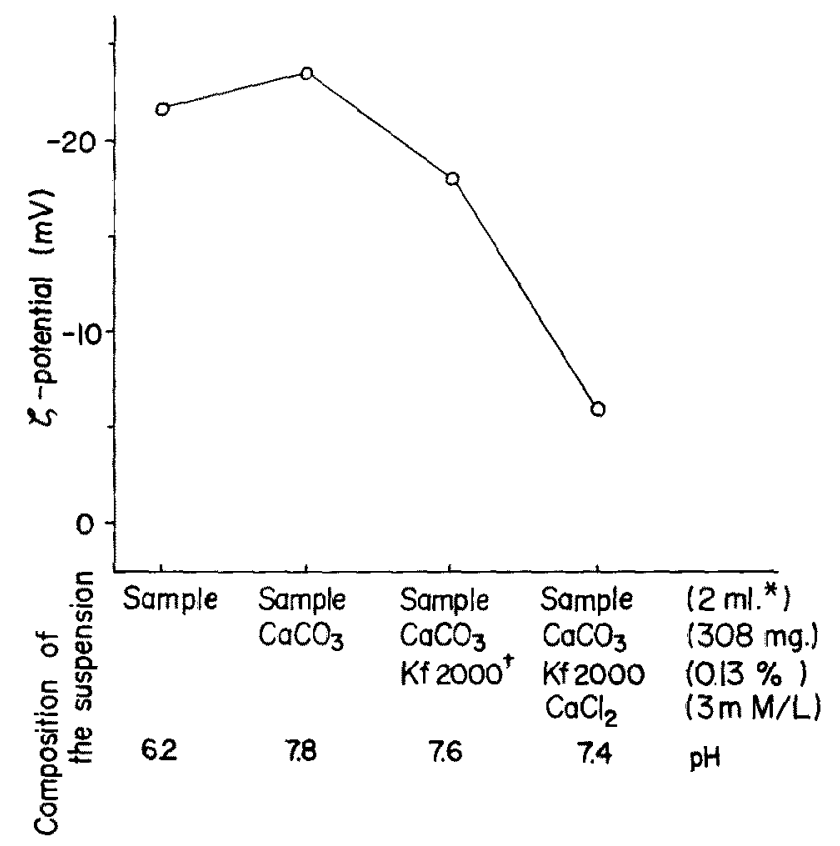

Fig. 7. 5-Potential of the particles at various combination of reagents added to the suspension. *Amount of the stock sample. Konanfloc 2000.

were greatest when calcium carbonate, Konanfloc 2000 and calcium chloride were all added to the suspension, indicating that this combination of reagents was favorable for formation of gallstone-like concrements in vitro. With this combination of the reagents added, i.e., at the favorable condition for coagulation, $\mathrm{pH}$ of the suspension was in a range from neutral to slightly alkaline reaction.

In formation of gallstone-like concrements in vitro, the velocity and the duration of rotation are presumed to significantly influence the results. Changing the rate of rotation, it was revealed that a rapider rotation generally resulted in smaller concrements and a slower rotation in larger ones. Although the effect of duration of rotation was not positively examined in this series of experiment, considerably large concrements were formed within 30 minutes on most occasions.

The gallstone-like coagulates produced in vitro by the method mentioned above were yellowish brown or umber in color. They were so compact in texture that one could pick them up with forceps. When allowed to stand on a sheet of filter paper, the coagulates were readily dried' up and the products resembled in appearance the genuine calcium bilirubinate stones. Fig. 8 presents a photogram of the gallstone-like concrements produced in vitro in the present experiments. 


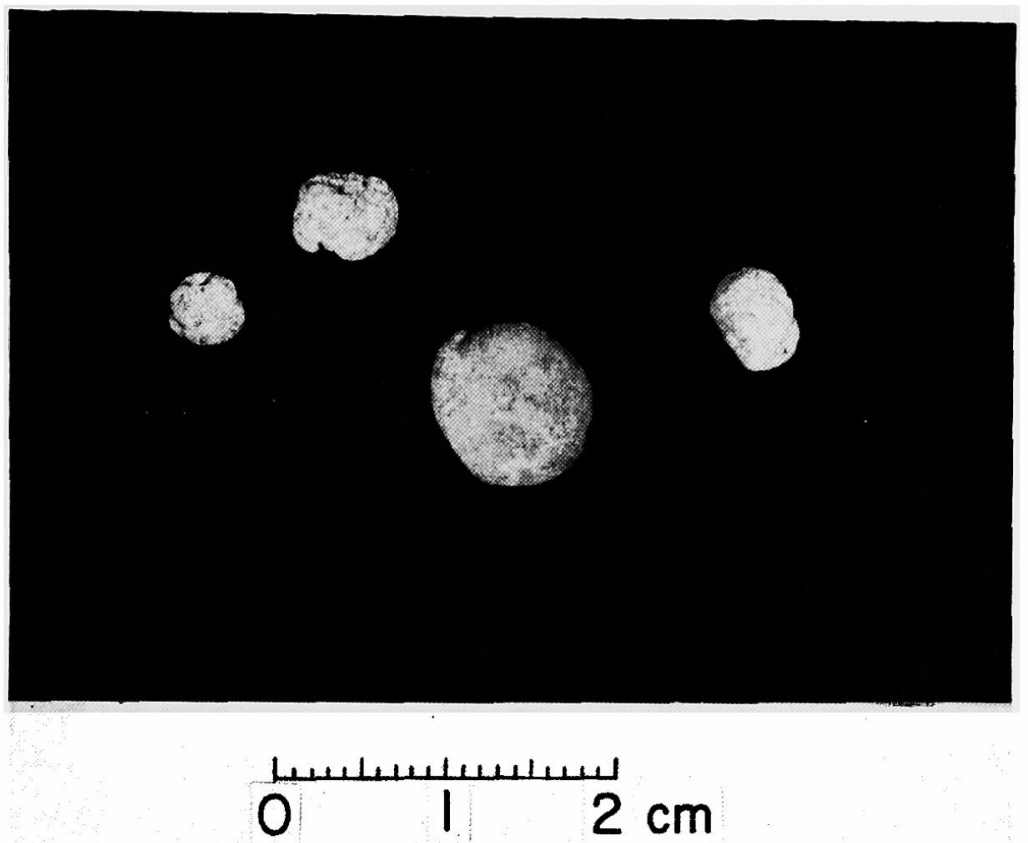

Fig. 8. Gallstone-like concrements produced in vitro from sediments of bile of patients with calcium bilirubinate stones.

\section{DISCUSSION}

The sediment of $\mathrm{T}$ drain bile obtained from patients with calcium bilirubinate stones consists mainly of calcium bilirubinate and there may be no doubt that this type of gallstones are formed as a result of coagulation and solidification of such a sediment. The problem to be solved is by what mechanism such coagulation and solidification occur in bile.

Generally speaking, solid particles dispersed in an aqueous solution induce imbalance of the ionic distribution at the solid-liquid interface; the surface of the particle is positively or negatively charged, according to the kind of the dispersed solid substance and to the ionic composition of the liquid phase, and the solution in the vicinity of the surface of the particle is charged oppositely, thus forming an electrical double layer. The charge distribution on the solution side is made up of an adsorbed layer of charge (Stern layer) and the diffuse space charge. A schematic representation of the charge and potential distribution in the electrical double layer is given in Fig. 9. It is generally accepted that the $\zeta$ potential, which is experimentally measurable, is approximately equal to the potential of the adsorbed layer.

When the suspending particles with electrical double layers are approximat- 


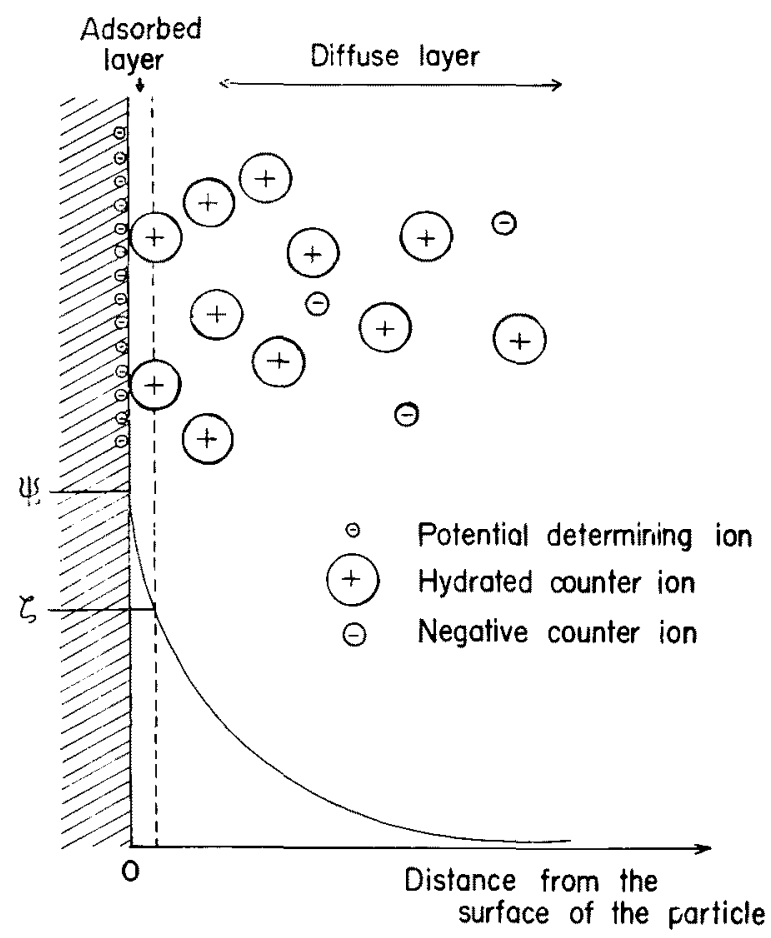

Fig. 9. Schematic representation of the structure of the electrical double layer and the distribution of electrostatic potential. $\Psi_{0}$, surface potential; $\zeta$, zeta-potential.

ed together by brownian movement, they repulse one another by the interaction of electrical double layers. At the same time, the particles are effected by van der Waals force that acts to attract them. Therefore, the actual interaction between two charged particles is represented by a resultant of repulsive potential energy $V_{R}$ and attractive or van der Waals potential energy $V_{A}$, as a function of the distance between the particles. As shown in Fig. 10, the resultant or total potential energy of this case exhibits a maximum $\left(\boldsymbol{V}_{n}\right)$ at a certain distance between the particles, and its value is determined by the surface potential of the particles, the thickness of the electrical double layer, etc. $V_{m}$ is a thermodynamic measure of the stability of a colloid solution or a suspension; the particles are dispersed if $V_{m}$ is larger than kinetic energy of the particles, whereas they are attracted and coagulate if $V_{m}$ is smaller than kinetic energy of the particles ${ }^{8}$. When an adequate electrolyte is added to a suspension, $V_{m}$ is lowered as a result of a decrease of $\zeta$-potential and a shrinkage of the ionic atmosphere, repulsive forces become overwhelmed by the effect of attractive energy and the particles coagulate. The coagulating effect of calcium chloride or aluminium 


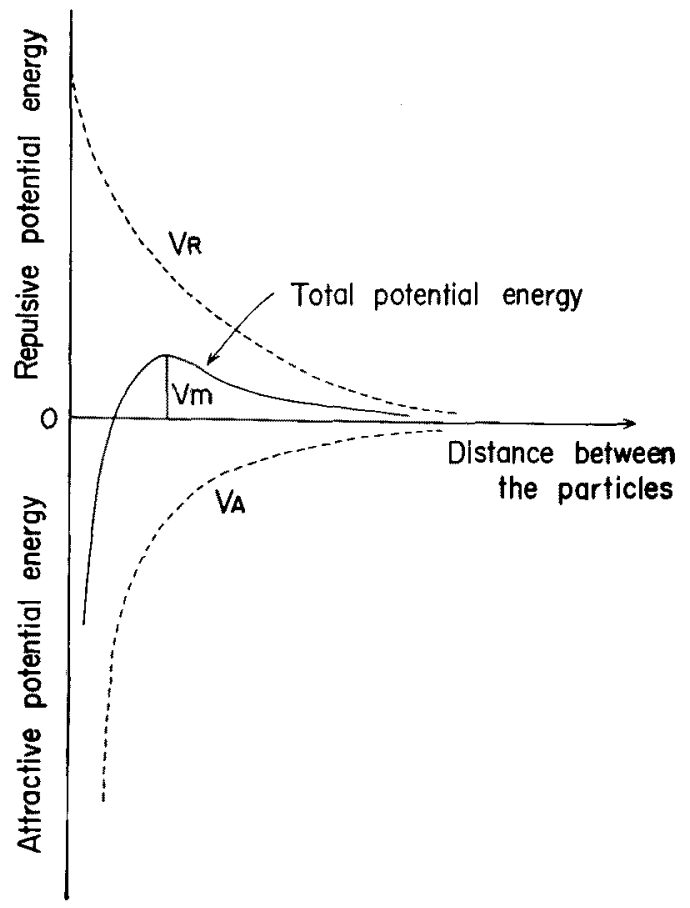

Fig. 10. Schematic representation of the potential energies existing in between two charged particles. $V_{R}$, repulsive potential energy; $V_{A}$, attractive or van der Waals potential energy; $V_{m}$, potential energy barrier.

chloride on the suspension of bile sediment may be explained by this theory.

The coagulate induced from bile sediment by addition of an inorganic electrolyte exhibited a strongly hydrophilic gel structure due to hydration of the particles. To approximate the particles more closely and to improve texture of the coagulate, the hydrated layer attached to the particles had to be removed by some measures. This was attained to some extent by addition to the suspension of calcium carbonate. The dehydrating effect of calcium carbonate may be in that the fine particles of this compound adsorb the colloid particles on their surfaces and thus disintegrate the gel structure of the flocks of the colloid particles. On the other hand, the effect of the linear high-molecular-weight organic compound, which also promoted coagulation of bile sediment, is probably ascribable to its remarkable adsorption characteristic and to a bridging action that is due to its fibrous structure ${ }^{9}$. In other words, it is presumed that such a compound has a property to adsorb colloid particles by functional groups of the molecule and, since a molecule has a large number of functional groups that are arranged around a linear skeleton, it can involve many colloid particles by bridging them. On this occasion, a polyelectrolyte with electric charge opposite to that of the 
colloid particles would be more effective than others because an electrostatic force due to the charge may enhance adsorption of the compound to the colloid particles, consequently lowering the $\zeta$-potential of the system. In fact, Konanfloc 1250 and Konanfloc 2000, which are polyelectrolytes of positive charge, proved to be most effective of the high-molecular-weight organic substances examined as far as coagulation of the particles of bile sediment, that were charged negatively, was concerned.

The coagulate of bile sediment thus prepared by addition of calcium carbonate, an inorganic electrolyte (e.g. calcium chloride) and an organic polyelectrolyte of positive charge (e.g. Konanfloc 2000) was still not firm enough to be comparable to a genuine gallstone. However, when such a coagulate was further subjected to a slow rotating movement of long duration, solid concrements resembling gallstones were eventually yielded. Although the role of rotation in the formation of gallstone-like concrements has not yet been completely elucidated, a probable interpretation may be that an adequate form of energy must be supplied from outside to overwhelm the potential energy barrier $V_{m}$ that may increase with an increase in the size of the particles as coagulation progresses. The authors thus succeeded in producing gallstone-like concrements in vitro from a suspension of particles prepared from infected human bile.

From the theoretical as well as experimental facts revealed in this study, the mechanism of formation of calcium bilirubinate stones in vivo may be explained as follows: When biliary flow becomes stagnant and bile is infected, a change is induced in the chemical composition of bile by the activity of bacterial $\beta$ glucuronidase and by some other causes, and calcium bilirubinate is separated from bile associated with other constituents of bile. Although the separated substances first form stable lyophobic colloids and are suspended in bile, the stability is soon decreased by the effect of inorganic and organic substances contained in bile and the colloids are sedimented as flocks. The flocks then gradually change into firm gallstones under the influence of exogenous kinetic energy that is exerted by movement of the gallbladder, bile flow due to secretory pressure of the liver, change of the body position and so on. The function of the biliary system to absorb water may participate, in part, in the solidification mechanism of gallstones.

The present experiment was conducted mainly for the purpose of clarifying the coagulation mechanism of calcium bilirubinate stones. However, the above view is expected to be applicable, to some extent, to the coagulation mechanism of other substances separated from a colloidal solution in the living organism. The authors would propose to name the above concept as "Solidification Theory by Maki and Suzuki". 


\section{Acknowledgment}

The authors wish to express their cordial thanks to Prof. T. Yamasaki and Assistant Prof. S. Usui, Research Institute of Mineral Dressing and Metallurgy, Tohoku University, and to Assistant Prof. M. Yusa, Department of Mechanical Engineering, Akita University, for their valuable suggestions given during the course of this study. The expense of this study was defrayed, in part, by the Ministry of Education of Japan through a grant from Scientific Research Fund, which is also gratefully acknowledged.

\section{References}

1) Maki, T., Sato, To. \& Sato, Ta., Tohoku J. exp. Med., 1962, 77, 179.

2) Saitoh, T., ibid., 1964, 83, 127.

3) Usui, S., Bull. Res. Inst. Min. Dress. and Met., Tohoku Univ. (Jap.), 1961, 17, 87.

4) Maki, T., Sato, T. \& Suzuki, N., Tohoku J. exp. Med., 1964, 82, 117.

5) Kruyt, H.R., Colloid Science, Vol. 1, Amsterdam, 1952.

6) Ohyama, T., Shimoizaka, J. \& Usui, S., Tohoku Kozan (Jap.), 1958, 4, 21.

7) Yusa, M. \& Gandin, A.M., Am. Ceram. Soc. Bull., 1964, 43, 402.

8) Verwey, E.J.W. \& Overbeek, J. Th. G., Theory of the Stability of Lyophobic Colloids, Amsterdam, 1948.

9) Yamasaki, T., Bull. Res. Inst. Min. Dress. and Met., Tohoku Univ. (Jap.), 1960, 16, 147. 